\title{
Kramers doublets, phonons, crystal-field excitations, and their coupling in $\mathrm{Nd}_{2} \mathrm{ZnIrO}_{6}$
}

\author{
Birender Singh $\odot,{ }^{1, *}$ M. Vogl, ${ }^{2}$ S. Wurmehl $\odot,{ }^{2,3}$ S. Aswartham $\odot,{ }^{2}$ B. Büchner, ${ }^{2,3}$ and Pradeep Kumar ${ }^{1, \dagger}$ \\ ${ }^{1}$ School of Basic Sciences, Indian Institute of Technology Mandi, Mandi-175005, India \\ ${ }^{2}$ Leibniz-Institute for Solid State and Materials Research, IFW-Dresden, 01069 Dresden, Germany \\ ${ }^{3}$ Institute of Solid-State Physics, TU Dresden, 01069 Dresden, Germany
}

(Received 25 February 2020; accepted 9 April 2020; published 12 May 2020)

\begin{abstract}
We report comprehensive Raman-scattering measurements on a single crystal of double-perovskite $\mathrm{Nd}_{2} \mathrm{ZnIrO}_{6}$ in a temperature range of 4-330 K, spanning a broad spectral range from 20 to $5500 \mathrm{~cm}^{-1}$. The paper focuses on lattice vibrations and electronic transitions involving Kramers doublets of the rare-earth $\mathrm{Nd}^{3+}$ ion with local $C_{1}$ site symmetry. Temperature evolution of these quasiparticle excitations has allowed us to ascertain the intricate coupling between lattice and electronic degrees of freedom in $\mathrm{Nd}_{2} \mathrm{ZnIrO}_{6}$. Strong coupling between phonons and crystal-field excitation is observed via renormalization of the self-energy parameter of the phonons, i.e., peak frequency and linewidth. The phonon frequency shows abrupt hardening and linewidth narrowing below $\sim 100 \mathrm{~K}$ for the majority of the observed first-order phonons. We observed splitting of the lowest Kramers doublets of ground state $\left({ }^{4} I_{9 / 2}\right)$ multiplets, i.e., lifting of the Kramers degeneracy, prominently at low temperature (below $\sim 100 \mathrm{~K}$ ), attributed to the $\mathrm{Nd}-\mathrm{Nd} / \mathrm{Ir}$ exchange interactions and the intricate coupling with the lattice degrees of freedom. The observed splitting is of the order of $\sim 2-3 \mathrm{meV}$ and is consistent with the estimated value. We also observed a large number of high-energy modes, 46 in total, attributed to the intraconfigurational transitions between $4 f^{3}$ levels of $\mathrm{Nd}^{3+}$ coupled to the phonons reflected in their anomalous temperature evolution.
\end{abstract}

DOI: 10.1103/PhysRevResearch.2.023162

\section{INTRODUCTION}

The physics of correlated electron systems associated with $5 d$ transition-metal oxides, especially Ir oxides, has drawn considerable research interest in recent years owing to the possible formation of quantum spin liquids, Mott insulators, unconventional superconductors, and Weyl semimetals [1-10]. Iridates provide an interesting interplay between strong spinorbit coupling and electron correlation due to their comparable strength along with exotic quasiparticle excitations such as orbitons [11]. Double-perovskite iridium-based materials of $A_{2} B \mathrm{IrO}_{6}$ crystal structure are interesting as well as challenging due to the freedom of tuning the quantum magnetic ground state with the choice of different magnetic and nonmagnetic ions on $A$ and $B$ crystallographic sites. So far, a majority of the studies have focused on tuning the magnetic exchange interactions by replacing $B$ sites with magnetic (i.e., Mn, Fe, Co, $\mathrm{Ni}$, and $\mathrm{Cu}$ ) and nonmagnetic (i.e., $\mathrm{Y}, \mathrm{Mg}$, and $\mathrm{Zn}$ ) elements [12-20]. The evolution of exotic quantum magnetic properties with the choice of the $A$-site element in these materials has not been extensively explored [21,22]. The substitution on the $A$ site with different lanthanide rare-earth elements may provide another degree of freedom to give insight into the

\footnotetext{
*birender.physics5390@gmail.com

†pkumar@iitmandi.ac.in

Published by the American Physical Society under the terms of the Creative Commons Attribution 4.0 International license. Further distribution of this work must maintain attribution to the author(s) and the published article's title, journal citation, and DOI.
}

complex ground state in these $5 d$ materials. This replacement may cause substantial local structural changes (i.e., change of bond distance Ir-O, bond angle $\mathrm{O}-\mathrm{Ir}-\mathrm{O}$, and rotation and/or tilting of $\mathrm{IrO}_{6}$ octahedra) within the unit cell, and may result in significant modulation of the strength of exchange interactions between nearest neighbors. Depending on the ionic radius of $A$-site ions, different quantum magnetic spin interactions such as Heisenberg and Kitaev type may be realized in doublepervoskite iridates [23-25]. Another important aspect of the presence of rare-earth ion in these systems is the crystal-field excitations (CFEs) originating from the splitting of rare-earth ion multiplets with specific local site symmetry owing to the surrounding ions static field. Probing these electronic excitations is very important because CFE as a function of temperature may provide crucial information about the nature of underlying ground state ordering and also provide the avenues to probe the coupling with lattice degrees of freedom [26-29].

The magnetic phase of $\mathrm{Nd}_{2} \mathrm{ZnIrO}_{6}$ is rather mysterious. It shows an antiferromagnetic ordering at $\sim 17 \mathrm{~K}$ attributed to the ordering of $\mathrm{Nd}^{3+}$ ions with a small contribution from the Ir sublattice, along with a broad transition at $8 \mathrm{~K}$. However, under weak magnetic field, a complex magnetic phase is reported with multiple magnetic transitions attributed to the interplay between $\mathrm{Nd}$ and Ir magnetic sublattices. The specific heat study of $\mathrm{Nd}_{2} \mathrm{ZnIrO}_{6}$ crystals also shows a sharp peak at $16.5 \mathrm{~K}$ and this magnetic transition peak is observed to exhibit a strong field dependence (significant suppression and broadening of magnetic transition peak) [22]. The $\mathrm{Nd}^{3+}$ ion here inhabits an eightfold coordinated $A$ site with $C_{1}$ local site symmetry that shows there is no symmetry element except identity. The sensitivity of the $\mathrm{Nd}^{3+}$ crystal-field levels to 
TABLE I. Wyckoff positions of the different atoms in the unit cell and irreducible representations of phonon modes of the monoclinic $\left(P 2_{1} / n\right.$; space group No. 14) double-perovskite $\mathrm{Nd}_{2} \mathrm{ZnIrO}_{6}$ at the $\Gamma$ point. $\mathrm{R}_{A g}$ and $\mathrm{R}_{B g}$ are the Raman tensors for $A_{g}$ and $B_{g}$ Raman active phonon modes, respectively. $\Gamma_{\text {total }}, \Gamma_{\text {Raman }}$, and $\Gamma_{\text {infrared }}$ represent the total normal modes, Raman, and infrared active modes, respectively.

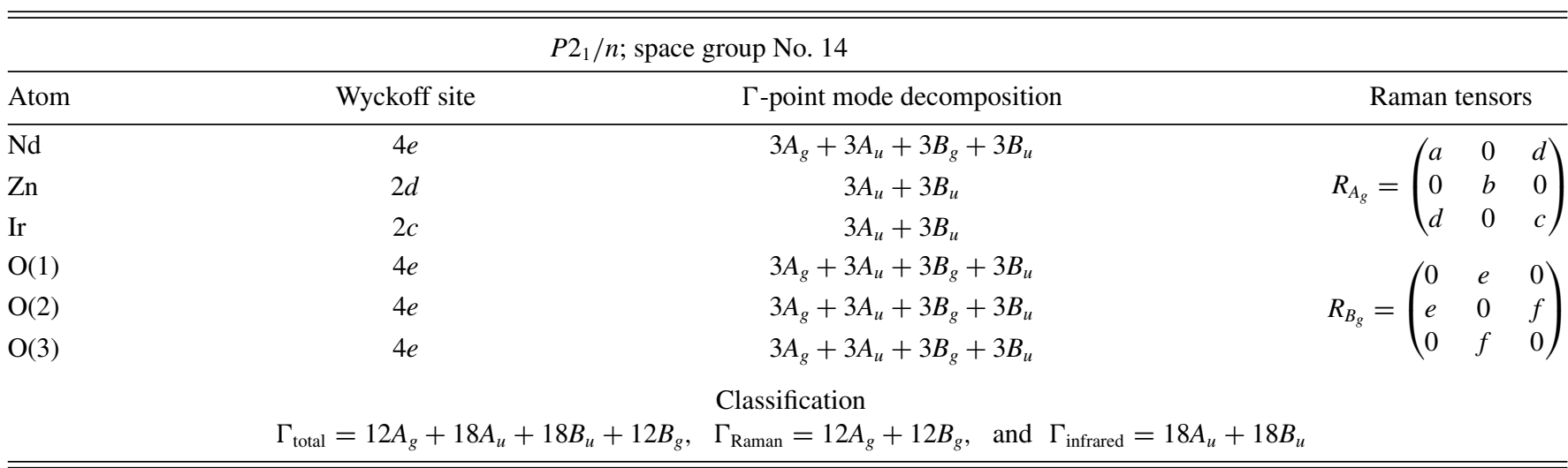

both local electric and magnetic fields makes Raman scattering by these CF levels a valuable probe for exploring the possible underlying interactions responsible for their ground state. Measurements of CFE are also important to understand various thermodynamical properties and accurately determine the generalized phonon density of states. Their measurement via Raman is complementary to complex neutron-scattering measurements, where extensive instrumentation is required [30]. Here, we present a detailed study of a single crystal of double-perovskite $\mathrm{Nd}_{2} \mathrm{ZnIrO}_{6}$ focusing on the phonons, $\mathrm{CFE}$, and the coupling between them. We note that not much attention has been paid for understanding the role of phonons and the coupling with other quasiparticle excitations in these Ir-based double-perovskite systems. We have performed a detailed lattice-dynamics study of $\mathrm{Nd}_{2} \mathrm{ZnIrO}_{6}$ in the temperature range of 4-330 K, and polarization-dependent Ramanscattering measurements along with the zone-centered phonon mode frequency calculations using first-principle based density functional theory (DFT). Our measurements reveal strong coupling between phonons and CFE, and evidence the lifting of Kramers degeneracy of $\mathrm{Nd}^{3+}$ ground state multiplets at low temperature attributed to the $\mathrm{Nd}-\mathrm{Nd}$ and $\mathrm{Nd}-\mathrm{Ir}$ exchange interactions.

\section{EXPERIMENTAL AND COMPUTATIONAL DETAILS}

Single crystals of $\mathrm{Nd}_{2} \mathrm{ZnIrO}_{6}$ were grown with the procedure described in detail in Ref. [22]; crystals were characterized with different structural and transport measurements [22]. The inelastic light (Raman) scattering measurements were performed on a single crystal of $\mathrm{Nd}_{2} \mathrm{ZnIrO}_{6}$ in backscattering configuration via a Raman spectrometer (LabRAM HR Evolution). We have employed 532-nm (2.33-eV) and 633-nm $(1.96-\mathrm{eV})$ lasers as an excitation probe for spectral excitation and the spectrometer is carefully calibrated for each laser excitation using a standard silicon crystal. The laser was focused on the sample surface using a $50 \times$ long working distance objective lens. A Peltier-cooled charge-coupled device (CCD) detector was used to collect the scattered light. Laser power was limited $(\leqslant 1 \mathrm{~mW})$ to avoid any local heating effects. Temperature-dependent measurements were performed with a closed-cycle He-flow cryostat (Montana Instruments), where the sample is mounted on top of the cold finger inside a chamber, which was evacuated to $1.0 \times 10^{-6}$ Torr, in the temperature range of $4-330 \mathrm{~K}$ with $\pm 0.1 \mathrm{~K}$ or better temperature accuracy.

Density functional theory based calculations were done to have insight about phonon dynamics using the plane-wave approach implemented in QUANTUM ESPRESSO [31]. The fully relativistic Perdew-Burke-Ernzerhof (PBE) was chosen as an exchange correlation functional within generalized gradient approximation to carry out these calculations. A plane-wave cutoff energy and charge density cutoff were set to 60 and $280 \mathrm{Ry}$, respectively. The numerical integration over the Brillouin zone was done with a $4 \times 4 \times 4 k$-point sampling mesh in the Monkhorst-Pack grid [32]. Dynamical matrix and zonecenter phonon frequencies were calculated using density functional perturbation theory, taking spin-orbit coupling without spin polarization into account [33]. All the calculations were performed with experimental lattice parameters reported in Ref. [22].

\section{RESULTS AND DISCUSSION}

\section{A. Raman scattering and zone-centered calculated phonon frequencies}

$\mathrm{Nd}_{2} \mathrm{ZnIrO}_{6}$ crystallizes in a monoclinic double-perovskite structure belonging to the $P 2_{1} / n$ space group (No. 14) and $C_{2 h}$ point group [22]. Within this structure the factor-group analysis predicts a total of 60 modes in the irreducible representation, out of which 24 are Raman active and 36 are infrared active (see Table I for details). The Raman spectra of $\mathrm{Nd}_{2} \mathrm{ZnIrO}_{6}$ single crystals were excited with two different incident photon energies, $633 \mathrm{~nm}(1.96 \mathrm{eV})$ and $532 \mathrm{~nm}$ $(2.33 \mathrm{eV})$. Figure 1 shows the Raman spectra of $\mathrm{Nd}_{2} \mathrm{ZnIrO}_{6}$ excited with a 633-nm laser at $4 \mathrm{~K}$, while the insets show its temperature evolution and comparison with $532 \mathrm{~nm}$ at 4 and $330 \mathrm{~K}$. We note that the Raman spectra when excited with a 532-nm laser exhibit extra features in the spectral range of $50-800 \mathrm{~cm}^{-1}$ and $1700-5500 \mathrm{~cm}^{-1}$, labeled as R1-R7 [see inset (2) of Fig. 1] and Q1-Q46 [see Figs. 6(a) and 6(b)], respectively. A detailed discussion about these modes is discussed in later sections. First, we will focus on the 


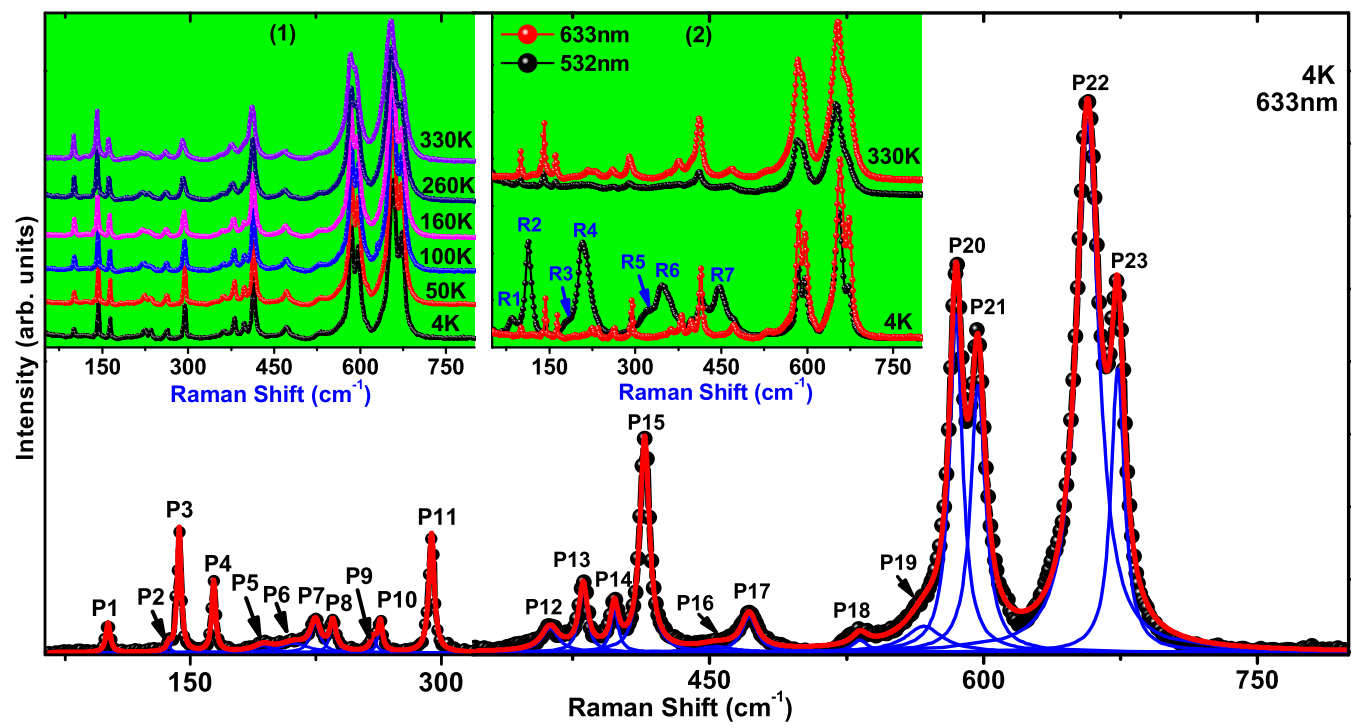

FIG. 1. Raman spectra of $\mathrm{Nd}_{2} \mathrm{ZnIrO}_{6}$ excited with a 633-nm laser. The solid thick red line indicates a total fit to the experimental data and the solid thin blue line corresponds to individual peak fitting, labels P1-P23 are phonon modes. Inset (1) shows the temperature evolution of the Raman spectra in the temperature range of 4-330 K excited with a 633-nm laser, and (2) shows the comparison of Raman spectra excited with 633- and 532-nm lasers at 4 and $330 \mathrm{~K}$; labels R1-R7 correspond to crystal-field modes.

first-order phonon modes shown in Fig. 1. We notice a total of 23 phonon modes in the spectrum excited with a 633-nm laser, labeled as P1-P23. The data are fitted with a sum of Lorentzian functions to obtain the spectral parameters such as phonon mode frequency $(\omega)$, linewidth $(\Gamma)$, and the integrated intensity.

In order to have insight on the specific vibration patterns of the observed phonon modes, we carried out zone-centered lattice-dynamics calculations using density functional perturbation theory. The calculated mode frequencies together with atomic displacements (vibrations) associated with particular phonon modes of $\mathrm{Nd}_{2} \mathrm{ZnIrO}_{6}$ are listed in Table II. The calculated phonon mode frequencies are found to be in very good agreement with the experimentally observed values. Figure 2 illustrates the schematic representations of the calculated eigenvectors of the corresponding phonon modes. The evaluation of these eigenvectors reveals that the low-frequency phonon modes P1-P3 and P5 are mainly attributed to the atomic displacement of $\mathrm{Nd}$ atoms, while $\mathrm{P} 4$ is composed of the displacement of $\mathrm{Nd} / \mathrm{Zn} / \mathrm{Ir}$. The high-frequency phonon modes P6-P23 correspond to the stretching and bending of the $\mathrm{Zn} / \mathrm{Ir}-\mathrm{O}$ bonds in the $\mathrm{Zn} / \mathrm{IrO}_{6}$ octahedra. The phonon modes assignment is done in accordance with our density functional theory calculations and polarization-dependent Raman measurements.

\section{B. Temperature and polarization dependence of the first-order phonon modes}

Figure 3 shows the temperature dependence of the frequency and linewidth of the first-order phonon modes of $\mathrm{Nd}_{2} \mathrm{ZnIrO}_{6}$ excited with a 633-nm laser. The following important observation can be made: (i) Low-energy phonon mode P1 $\left(\sim 100 \mathrm{~cm}^{-1}\right)$ exhibits phonon softening below $\sim 100 \mathrm{~K}$; however, no anomaly is observed in the linewidth of this mode in the entire temperature range. (ii) Interestingly, the phonon modes P3, P4, P7, P8, P10, P11, P15, P21, and P22 show significant frequency hardening with a decrease in temperature below $\sim 100 \mathrm{~K}$ down to the lowest recorded temperature, while P20 and P23 phonon modes display a slight softening. Additionally, a clear change in the linewidths of all these phonon modes is observed around $\sim 100 \mathrm{~K}$, i.e., anomalous line narrowing below this temperature. Similar temperature dependence is also observed for the frequency and linewidth of phonon modes excited with a 532-nm laser (not shown here). We note that the anomalies are more pronounced for the low-frequency phonon modes. The observed anomaly in the phonon mode frequencies and linewidths at low temperature clearly indicate strong interaction of the phonons with other degrees of freedom. Long-range magnetic ordering in this system is reported at $\sim 17 \mathrm{~K} \mathrm{[22]}$; however, the phonon anomalies are observed at temperatures as high as $\sim 100 \mathrm{~K}$, well above the magnetic transition temperature. This observation suggests its origin from degrees of freedom in the observed phonon anomalies other than magnetic ones, such as electronic degrees of freedom. Here, a coupling to crystal-field multiplets may be at work.

To understand the temperature dependence of the firstorder phonon modes, we have fitted the phonon mode frequency and linewidth from $\sim 100$ to $330 \mathrm{~K}$ with an anharmonic phonon-phonon interaction model including both three- and four-phonon decay channels given as [34]

$\omega(T)=\omega_{0}+A\left(1+\frac{2}{e^{x}-1}\right)+B\left[1+\frac{3}{e^{y}-1}+\frac{3}{\left(e^{y}-1\right)^{2}}\right]$,

and

$\Gamma(T)=\Gamma_{0}+C\left(1+\frac{2}{e^{x}-1}\right)+D\left[1+\frac{3}{e^{y}-1}+\frac{3}{\left(e^{y}-1\right)^{2}}\right]$, 
TABLE II. List of DFT-based calculated phonon frequencies and the experimentally observed first-order phonon mode frequencies of $\mathrm{Nd}_{2} \mathrm{ZnIrO}_{6}$ at $4 \mathrm{~K}$ with a $633-\mathrm{nm}$ excitation laser and fitting parameters obtained by using equations as described in the text. Mode assignment is done in accordance with polarization-dependent study and lattice-dynamics calculations. Units are in $\mathrm{cm}^{-1}$.

\begin{tabular}{|c|c|c|c|c|c|c|c|c|}
\hline \multirow[b]{2}{*}{ Mode assignment } & \multirow[b]{2}{*}{ Expt. $\omega(4 \mathrm{~K})$} & \multirow[b]{2}{*}{ DFT $\omega$} & \multicolumn{6}{|c|}{ Fitted Parameters } \\
\hline & & & $\omega_{0}$ & A & B & $\Gamma_{0}$ & $\mathrm{C}$ & $\mathrm{D}$ \\
\hline $\mathrm{P} 1: A_{g}(\mathrm{Nd})$ & 100.7 & 93.6 & $101.4 \pm 0.2$ & $-0.01 \pm 0.03$ & $-0.003 \pm 0.001$ & $3.2 \pm 0.6$ & $0.06 \pm 0.02$ & $0.003 \pm 0.001$ \\
\hline P2: $B_{g}(\mathrm{Nd})$ & 137.1 & 124.0 & & & & & & \\
\hline $\mathrm{P} 3: A_{g}(\mathrm{Nd})$ & 143.3 & 131.2 & $142.5 \pm 0.2$ & $-0.16 \pm 0.11$ & $-0.003 \pm 0.002$ & $2.8 \pm 0.2$ & $0.15 \pm 0.08$ & $0.005 \pm 0.002$ \\
\hline $\mathrm{P} 4: B_{g}(\mathrm{Nd}, \mathrm{Zn}, \mathrm{Ir})$ & 163.9 & 131.9 & $163.8 \pm 0.3$ & $-0.26 \pm 0.13$ & $-0.009 \pm 0.003$ & $2.4 \pm 0.3$ & $0.26 \pm 0.18$ & $0.008 \pm 0.005$ \\
\hline P5: $B_{g}(\mathrm{Nd})$ & 193.6 & 150.5 & & & & & & \\
\hline P6: $B_{g}(\mathrm{O})$ & 211.6 & 207.0 & & & & & & \\
\hline $\mathrm{P} 7: B_{g}(\mathrm{O})$ & 224.7 & 220.9 & $223.4 \pm 0.6$ & $-1.18 \pm 0.07$ & $-0.007 \pm 0.004$ & $7.2 \pm 3.4$ & $2.71 \pm 1.13$ & $-0.004 \pm 0.002$ \\
\hline $\mathrm{P} 8: A_{g}(\mathrm{O})$ & 235.2 & 234.1 & $234.0 \pm 1.2$ & $-0.49 \pm 0.05$ & $-0.017 \pm 0.003$ & $8.2 \pm 1.8$ & $0.27 \pm 0.13$ & $0.039 \pm 0.012$ \\
\hline P9: $B_{g}(\mathrm{O})$ & 259.4 & 238.8 & & & & & & \\
\hline P10: $A_{g}(\mathrm{O})$ & 263.5 & 276.1 & $262.7 \pm 0.5$ & $-0.46 \pm 0.27$ & $-0.015 \pm 0.001$ & $4.0 \pm 0.7$ & $0.92 \pm 0.41$ & $0.023 \pm 0.017$ \\
\hline $\mathrm{P} 11: A_{g}(\mathrm{O})$ & 294.2 & 279.3 & $294.7 \pm 0.4$ & $-1.09 \pm 0.34$ & $-0.02 \pm 0.01$ & $3.6 \pm 0.7$ & $1.18 \pm 0.51$ & $0.064 \pm 0.032$ \\
\hline P12: $A_{g}(\mathrm{O})$ & 362.5 & 328.5 & & & & & & \\
\hline P13: $B_{g}(\mathrm{O})$ & 380.7 & 357.0 & $382.4 \pm 0.6$ & $-2.29 \pm 0.59$ & $-0.03 \pm 0.02$ & $4.8 \pm 2.3$ & $1.99 \pm 0.54$ & $0.234 \pm 0.146$ \\
\hline $\mathrm{P} 14: A_{g}(\mathrm{O})$ & 397.8 & 372.6 & & & & & & \\
\hline P15: $B_{g}(\mathrm{O})$ & 414.4 & 388.8 & $415.2 \pm 0.3$ & $-0.91 \pm 0.31$ & $-0.07 \pm 0.02$ & $4.9 \pm 1.0$ & $1.67 \pm 0.67$ & $0.168 \pm 0.071$ \\
\hline P16: $B_{g}(\mathrm{O})$ & 453.2 & 423.4 & & & & & & \\
\hline P17: $B_{g}(\mathrm{O})$ & 471.7 & 441.4 & $471.7 \pm 1.5$ & $-0.32 \pm 0.11$ & $-0.24 \pm 0.13$ & $7.4 \pm 3.1$ & $3.53 \pm 1.57$ & $0.113 \pm 0.021$ \\
\hline P18: $A_{g}(\mathrm{O})$ & 532.3 & 538.3 & & & & & & \\
\hline P19: $B_{g}(\mathrm{O})$ & 567.3 & 541.6 & & & & & & \\
\hline P20: $B_{g}(\mathrm{O})$ & 584.9 & 558.0 & $587.7 \pm 0.6$ & $-2.32 \pm 0.76$ & $-0.04 \pm 0.01$ & $6.0 \pm 2.56$ & $2.71 \pm 0.91$ & $0.515 \pm 0.211$ \\
\hline P21: $A_{g}(\mathrm{O})$ & 597.0 & 582.1 & $601.1 \pm 1.3$ & $-4.20 \pm 1.38$ & $-0.03 \pm 0.01$ & $6.3 \pm 2.93$ & $4.62 \pm 2.31$ & $-0.088 \pm 0.037$ \\
\hline P22: $A_{g}(\mathrm{O})$ & 656.9 & 668.4 & $660.4 \pm 1.4$ & $-3.56 \pm 1.58$ & $-0.27 \pm 0.19$ & $7.63 \pm 3.90$ & $8.73 \pm 3.34$ & $0.378 \pm 0.171$ \\
\hline P23: $A_{g}(\mathrm{O})$ & 673.5 & 721.7 & $673.5 \pm 0.8$ & $0.71 \pm 0.18$ & $-0.33 \pm 0.11$ & $4.37 \pm 2.61$ & $5.64 \pm 2.34$ & $0.364 \pm 0.113$ \\
\hline
\end{tabular}

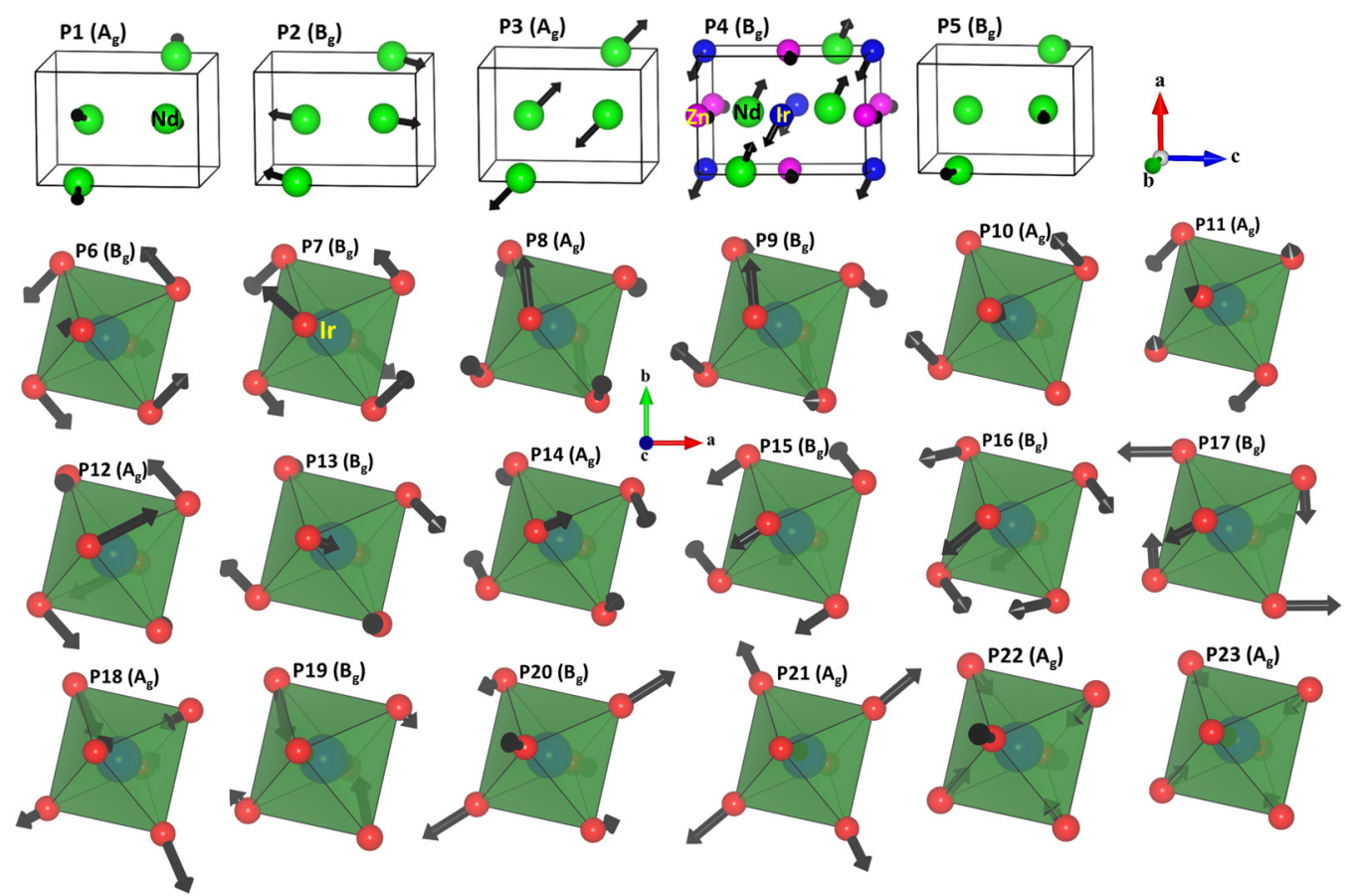

FIG. 2. Calculated eigenvectors of the phonon modes P1-P23, where green, blue, pink, and red spheres represents the Nd, Ir, Zn, and oxygen atoms, respectively. Black arrows on the atoms indicate the direction of atomic displacement and the arrow size illustrates the magnitude of atomic vibration. $a, b$, and $c$ indicate the crystallographic axes. 

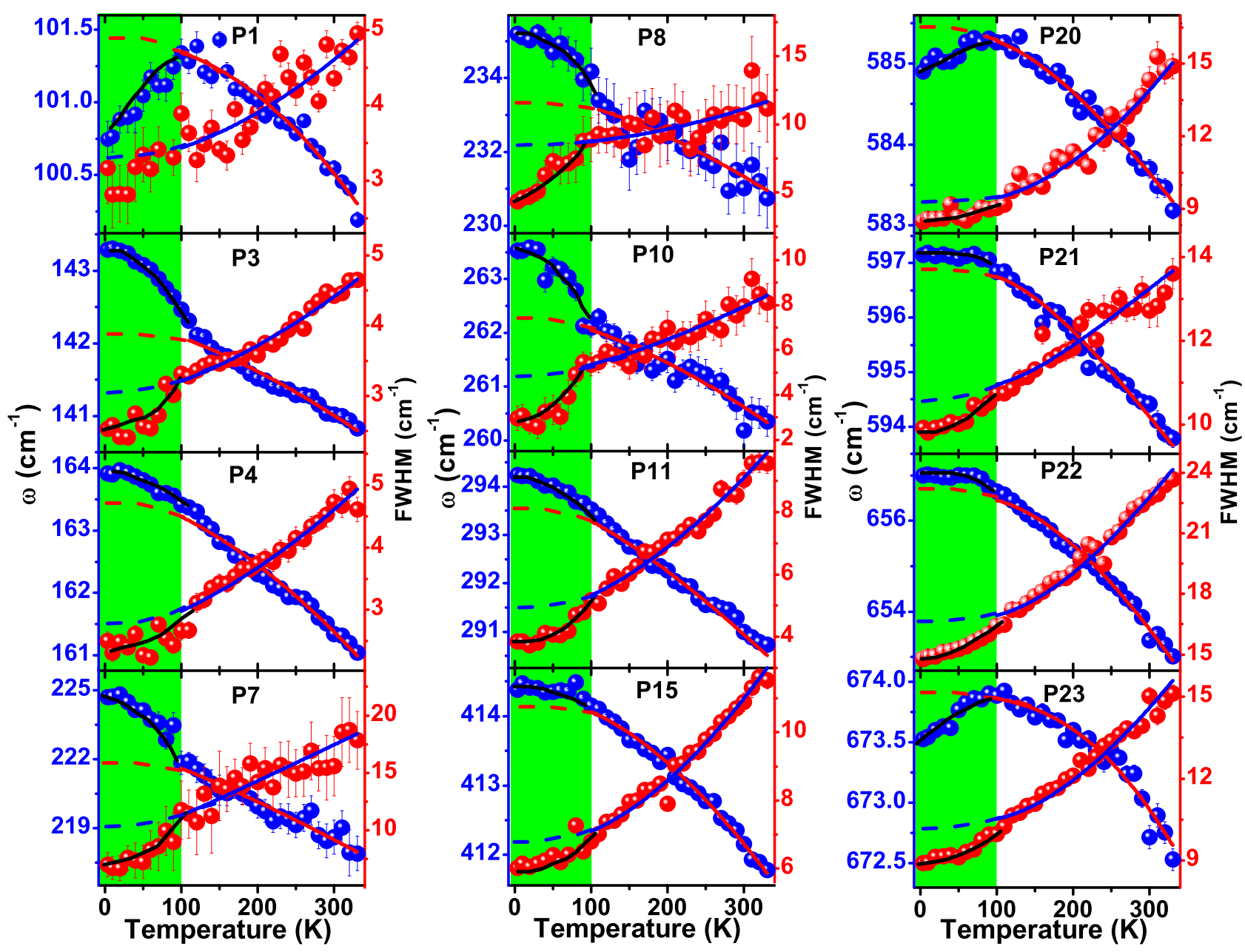

FIG. 3. Temperature dependence of the mode frequencies and linewidths of the first-order phonon modes P1, P3, P4, P7, P8, P10, P11, $\mathrm{P} 15$, and P20-P23. Blue and red spheres represent experimental value of frequencies and linewidths, respectively. Solid red and blue lines in temperature range are the fitted curves as described in the text. Solid black lines are a guide to the eye and broken lines are extended estimation of fitted curves. Shaded portion below $\sim 100 \mathrm{~K}$ depicts the region where pronounced phonon anomalies are observed.

where $\omega_{0}$ and $\Gamma_{0}$ are the mode frequency and linewidth at $T=$ $0 \mathrm{~K}$, respectively; $x=\frac{\hbar \omega_{0}}{2 k_{B} T}, y=\frac{\hbar \omega_{0}}{3 k_{B} T}$; and $A, B, C$, and $D$ are constants. The fitted parameters are summarized in Table II. The expected temperature variation of mode frequency and linewidth due to anharmonicity is shown as solid red and blue lines (see Fig. 3). Within the anharmonic interaction picture phonon modes are expected to become sharper and mode frequency gradually increases with decreasing temperature. Notably, at low temperature below $\sim 100 \mathrm{~K}$ the phonon mode self-energy parameters, i.e., mode frequencies and linewidths, exhibit significant deviation from the curve estimated by anharmonic phonon-phonon interaction model. The majority of the observed phonon mode frequencies harden anomalously below $\sim 100 \mathrm{~K}$, except modes P1, P20, and P23 as they exhibit phonon softening. The observed diametrically different nature of phonon renormalization, i.e., softening and hardening, may arise from the fact that different phonon modes involves different exchange paths and correlation effects depending on their symmetry and energy in addition to the fact that the magnitude of vibration of atoms involved is also different for different modes (see Fig. 2). Interestingly, the magnitude of the phonon frequency hardening is observed to be more pronounced for the low-frequency phonon modes (below $300 \mathrm{~cm}^{-1}$ ). In general, the magnitude of anomalous modulation of phonon frequencies is usually attributed to the interaction strength of a particular phonon mode with, e.g., spin degrees of freedom and the change in phonon mode frequency is related to the spin-spin correlation function given as [35] $\Delta \omega \approx \lambda\left\langle S_{i} S_{j}\right\rangle$, where $\lambda=\frac{\partial^{2} J_{i j}}{\partial u^{2}}$ is the spin-phonon coupling coefficient $\left(J_{i j}\right.$ is the superexchange coupling integral) and $\left\langle S_{i} S_{j}\right\rangle$ is the spin-spin correlation function. Hence, in a long-range magnetic phase the phonon renormalization mainly occurs due to the strong coupling of the lattice with spin degrees of freedom. The effect of magnetic degrees of freedom is expected to be minimal if not zero in this case because $T_{N}(\sim 17 \mathrm{~K})$ is much lower than the temperature $(\sim 100 \mathrm{~K})$ where anomalies are observed. In this scenario, the pronounced self-energy renormalization of the phonon modes implies a strong coupling of the lattice with electronic degrees of freedom via crystal-field excitation associated $4 f$ levels of the $\mathrm{Nd}^{3+}$ ion. Indeed, we found signature of strong coupling of lattice with crystal-field excitations arising from ground 

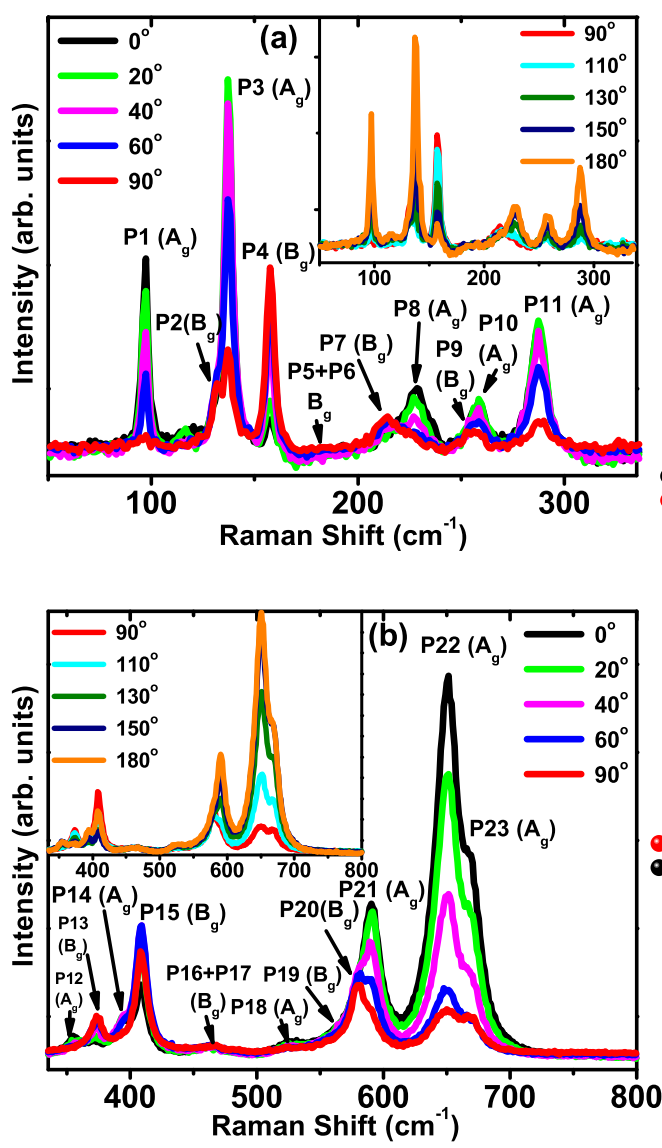
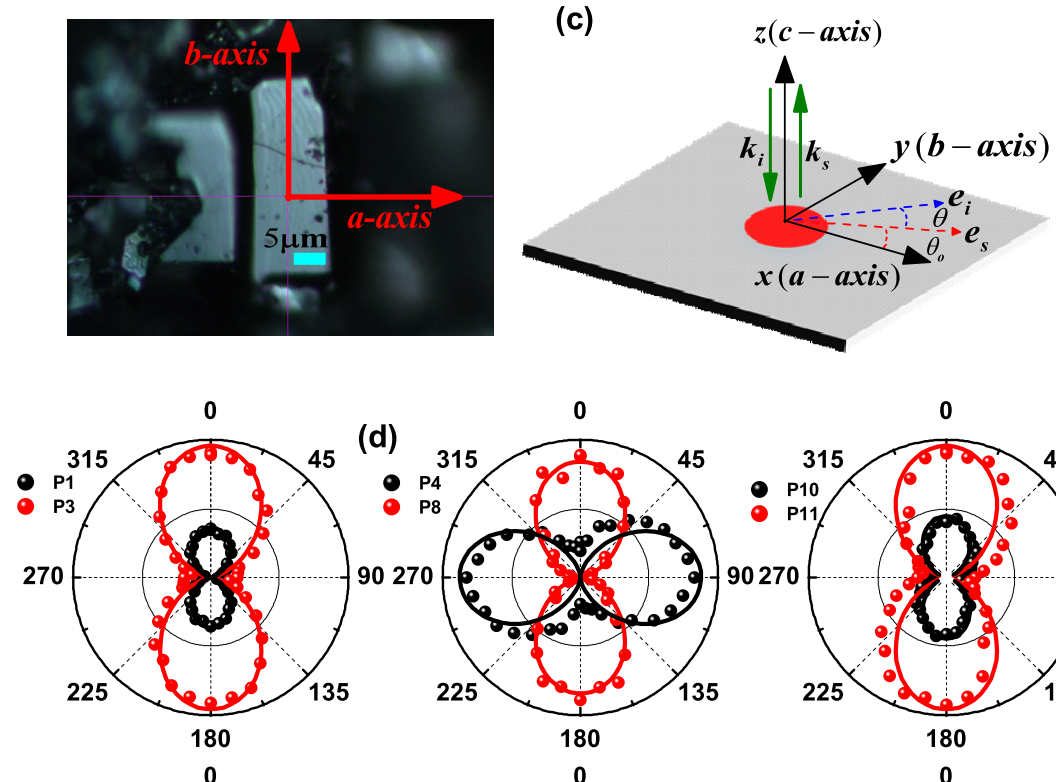

(d)


0
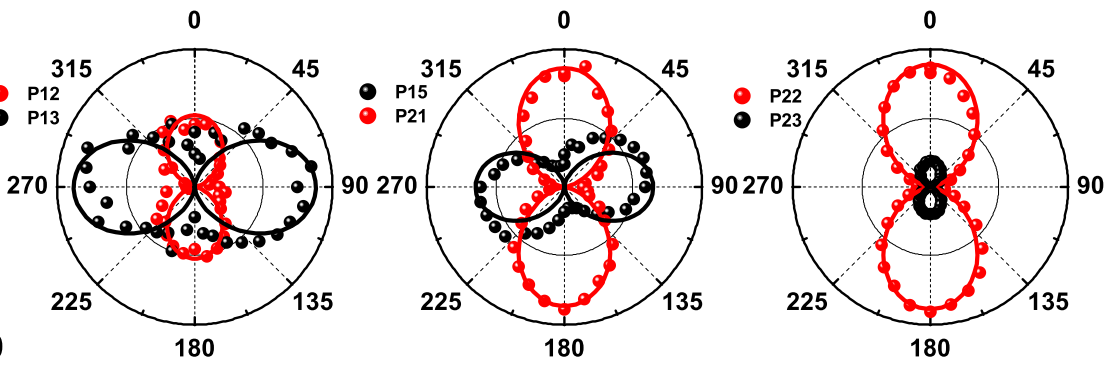

FIG. 4. Angular dependence of the Raman spectrum of $\mathrm{Nd}_{2} \mathrm{ZnIrO}_{6}$; (a), (b) show the raw data at different incident light angle in the range of $0^{\circ}-90^{\circ}$ (inset shows from $90^{\circ}$ to $180^{\circ}$ ) in the spectral range of $50-330 \mathrm{~cm}^{-1}$ and $330-800 \mathrm{~cm}^{-1}$, respectively. (c) Left side shows the optical micrograph of the microcrystal and right side shows the schematic depicting the direction of polarization of incident and scattered light in the $a b$ plane. $\theta_{o}$ is the angle between scattered light polarization vector $\left(\hat{e}_{s}\right)$ and crystal $a$ axis, and $\theta$ is the angle between $\hat{e}_{i}$ and $\hat{e}_{s}$. $k_{i}$ and $k_{s}$ represent the direction of incident and scattered beams. (d) Polar plots of the angular-dependence intensity of the prominent phonon modes. The solid curves are fitted with angular-dependence mode intensity equations as described in text.

state $\left({ }^{4} I_{9 / 2}\right)$ multiplets of $\mathrm{Nd}^{3+}$ ion at low temperature because of their same energy and symmetry, led to the strong renormalization of the phonon self energy parameters. However, we observed a little anomaly in some of the phonon mode frequency around $T_{N}$ and it may correspond to the coupling of the lattice with underlying magnetic degrees of freedom (see Fig. 3).

To decipher the symmetry of the phonon modes, we also performed polarization-dependent measurements. Polarization-dependent measurements using Raman spectroscopy can be done using equivalent configurations based on the polarization of incident or scattered light or rotation of the sample. In our polarization-dependent study, polarization of the incident laser light is varied; i.e., incident laser polarization is rotated using a polarizer with an increment of $10^{\circ}$ from $0^{\circ}$ to $360^{\circ}$, while the direction of the scattered light polarization is fixed using an analyzer. Figures 4(a) and 4(b) illustrate the polarization dependence of the Raman spectra of $\mathrm{Nd}_{2} \mathrm{ZnIrO}_{6}$ at different angles of the incident light polarization vector on the sample surface ranging from $0^{\circ}$ to $90^{\circ}$ in the spectral ranges of $50-330 \mathrm{~cm}^{-1}$ and $330-800 \mathrm{~cm}^{-1}$, respectively [the insets of
Figs. 4(a) and 4(b) show Raman spectra at different angles from $90^{\circ}$ to $180^{\circ}$ ]. Angular dependence of the intensity of the prominent phonon modes is shown in Fig. 4(d). Intensity of these prominent first-order phonon modes shows a twofold symmetric nature, i.e., having maximum intensity at $0^{\circ} / 90^{\circ}$ and $180^{\circ} / 270^{\circ}$. One may understand the observed variation in the intensity as a function of the polar angle within the semiclassical approach. As the incident and scattered polarized light lies in the $a b$ plane, the unit vector associated with the incident $\left(\hat{e}_{i}\right)$ and scattered $\left(\hat{e}_{s}\right)$ light polarization may be expressed in the decomposed form as $\left[\cos \left(\theta+\theta_{o}\right) \sin \left(\theta+\theta_{o}\right) \quad 0\right]$ and $\left[\cos \left(\theta_{o}\right) \sin \left(\theta_{o}\right) \quad 0\right]$, respectively, for the case of propagation of linear-polarized light in the $z$ direction [see right panel of Fig. 4(c)]. Within the semiclassical approach, the Raman-scattering cross section is given as $I_{\text {Raman }} \propto\left|\hat{e}_{s}^{t} \cdot R \cdot \hat{e}_{i}\right|^{2}$, where $t$ denotes the transpose of $\hat{e}_{s}$ and $R$ (see Table I) is the Raman tensor [36-38]. Using the above expression for the intensity, the angular dependence of Raman intensity for the modes $A_{g}$ and $B_{g}$ are given as $I_{A_{g}}=\left|a \cos \left(\theta_{o}\right)\left[\cos \left(\theta+\theta_{o}\right)+b \sin \left(\theta_{o}\right) \sin \left(\theta+\theta_{o}\right)\right]\right|^{2}$ and $\quad I_{B_{g}}=\left|e \cos \left(\theta_{o}\right)\left[\sin \left(\theta+\theta_{o}\right)+e \sin \left(\theta_{o}\right) \cos \left(\theta+\theta_{o}\right)\right]\right|^{2}$. Here $\theta_{o}$ is an arbitrary angle from the $a$ axis and is constant. 

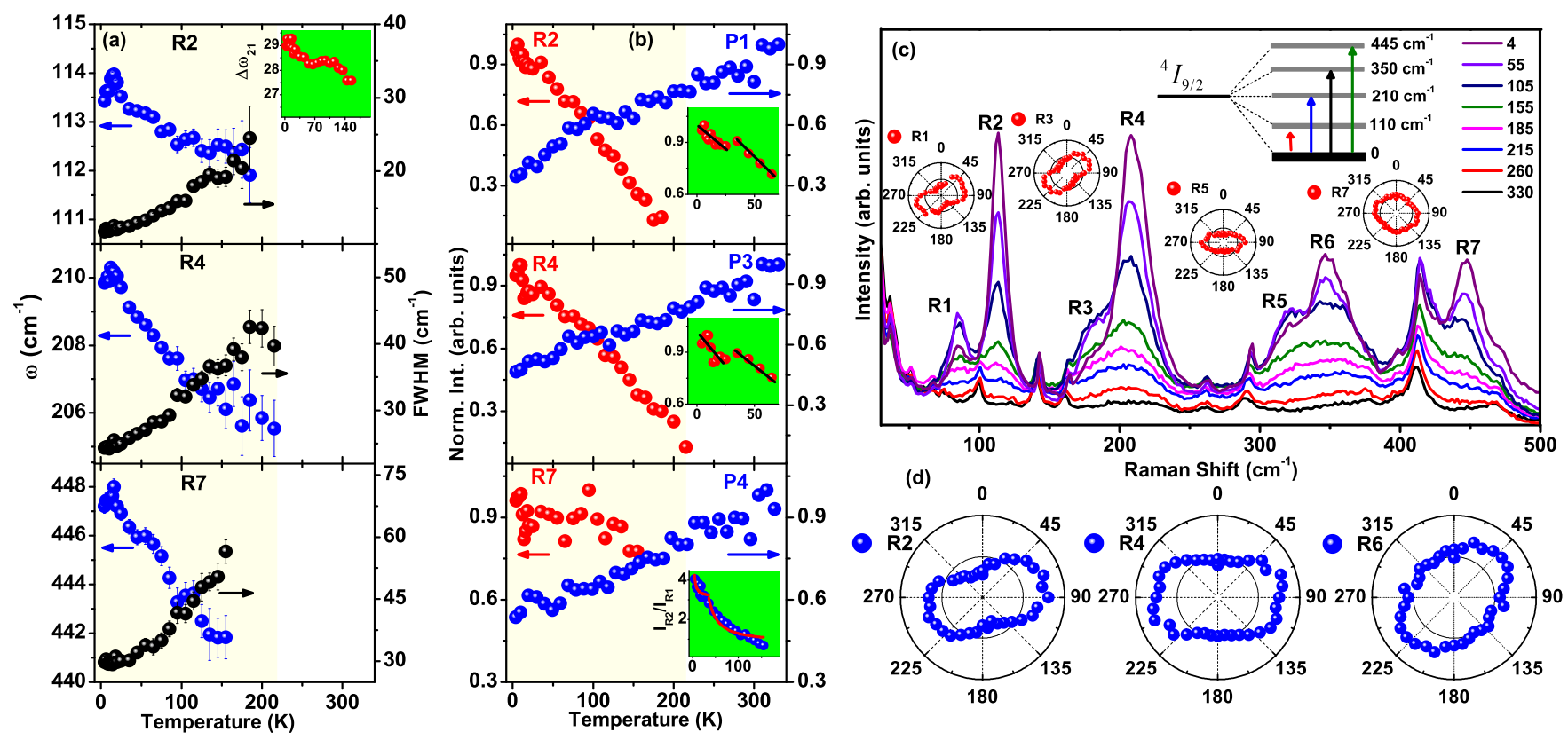

FIG. 5. (a) Temperature dependence of the frequency and linewidth of the prominent crystal-field modes R2, R4, and R7; inset of top panel shows frequency difference $\left(\Delta \omega_{21}=\omega_{2}-\omega_{1}\right)$ between modes R2 and R1 as a function of temperature. (b) Temperature dependence of the intensity of modes R2, R4, and R7 along with those of first-order phonon modes P1, P3, and P4. Insets in top and middle panels show the change in slope of the intensity near the magnetic transition temperature; solid lines are a guide to the eye. Inset in bottom panel shows the intensity ratio (i.e., R2/R1) and solid line is the fitted curve as described in the text. (c) Temperature evolution of the modes (R1-R7) in the spectral range of $20-500 \mathrm{~cm}^{-1}$ excited with a 532-nm laser. Inset shows the polarization dependence of the modes R1, R3, R5, and R7 at $4 \mathrm{~K}$, and a schematic for the CF splits levels of the $\mathrm{Nd}^{3+}$ ground state $\left({ }^{4} I_{9 / 2}\right)$ multiplets into five Kramers doublets. (d) Polarization dependence of $R_{2}, R_{4}$, and $R_{6}$ modes showing the quasi-isotropic nature.

Therefore, without the loss generality it may be chosen to be zero, giving rise to the expression for the Raman intensity as $I_{A_{g}}=|a|^{2} \cos ^{2}(\theta)$ and $I_{B_{g}}=|e|^{2} \sin ^{2}(\theta)$. From these intensity expressions it is clear that these two types of phonon modes are out of phase with each other; i.e., the angular dependence of the $A_{g}$ mode intensity decreases to zero when incident and scattered polarization vectors are perpendicular to each other. On the other hand, the intensity of the $B_{g}$ phonon mode shows maxima in this configuration. The solid lines in Fig. 4(d) represent the fitted curves using the above expression for the intensity of the phonon modes. The fitted curves are in very good agreement with the experimental angle variation of the mode intensities. Using the polarization dependence of the phonon modes and our DFT-based calculations, we have assigned the symmetry of the observed phonon modes (see Table II). Symmetry of the phonon modes extracted from our polarization-dependent measurement is mapped nicely with the mode symmetries calculated using the first-principle DFT-based calculations.

\section{Crystal-field excitations and coupling with phonons}

In addition to the phonon modes several additional modes are observed in the spectrum excited with a 532-nm $(2.33-\mathrm{eV})$ laser. First, we will discuss the modes observed in the lowfrequency range below $\sim 500 \mathrm{~cm}^{-1}$, labeled as R1-R7 [see Fig. 5(c) and inset (2) of Fig. 1], which appear in the lowtemperature regime below $\sim 150-200 \mathrm{~K}$. We have assigned these modes to the crystal-field excitations within the ground state multiplets of $\mathrm{Nd}^{3+}\left({ }^{4} I_{9 / 2}\right.$, ground state has five Kramers doublets; see inset of Fig. 5(c) for their energy levels [39,40]). As the frequencies of Raman active modes are independent of the incident photon energy, as a result they appear at the same position in Raman shift even with different energy of the excitation source; also, Raman active modes appear at the same position in both the Stokes and anti-Stokes sides of the spectrum. However, the emission or absorption peak arising due to transitions between different electronic levels will be shifted equal to the separation between the two incident laser energies, whereas their absolute position in energy must be preserved. These low-energy modes are visible in both the Stokes and anti-Stokes sides of the spectrum with a 532-nm laser at the same position suggesting the Raman active nature of these excitations.

Figures 5(a) and 5(b) show the temperature dependence of the frequency, linewidth, and intensity of the prominent modes, i.e., R2, R4, and R7. Shift in their frequency is quite significant and is as high as $\sim 3 \%$ (for R4) within a short interval of temperature suggesting intricate coupling with the phonon modes of similar energy and symmetry. The integrated intensity of these modes also increases at a very fast rate with decreasing temperature [see left side of Fig. 5(b)], which is typical for Raman scattering associated with crystal-field transitions (because the ground state population increases with decreasing temperature) and shows temperature evolution opposite to that of phonons [see right side of Fig. 5(b) showing intensity for modes P1, P3, and P4]. We also did the polarization dependence of these modes at low 
temperature [see Fig. 5(d) and inset of Fig. 5(c)]. The polarization dependence of these modes reflects their quasi-isotropic nature and is quite different from the observed phonon mode polarization behavior, confirming the nonphononic nature of these modes. From the symmetry analysis, CF levels of $\mathrm{Nd}^{3+}$ in the ground state (i.e., ${ }^{4} I_{9 / 2}$ ) multiplets split into five Kramers doublets. Since $\mathrm{Nd}^{3+}$ has a $C_{1}$ local site symmetry, all the levels have $A$ symmetry and transition between them are expected to be active in all polarizations [30]. Therefore, the observation of quasi-isotropic nature of the polarizationdependent behavior of these CF modes is in line as predicted by the symmetry analysis. We note that these lower-energy crystal-field modes appear only at low temperature (below $\sim 200 \mathrm{~K}$ ). The Raman cross section for the CFE is often weak and these have been observed generally with the strong coupling with nearby Raman active phonon modes via strong electron-phonon interaction. Two quasiparticle excitations of similar energy and symmetry are expected to couple strongly [30]; therefore a phonon mode and CFE of the same symmetry and similar energy are also expected to couple strongly. As discussed in the previous section above, renormalization of the phonon modes at low temperature $(\sim 100 \mathrm{~K})$ is very strong (see Fig. 3) and interestingly renormalization effects are more pronounced for low-energy phonon modes (e.g., P1, P3, P8, $\mathrm{P} 10$, and $\mathrm{P} 11$; all have $A_{g}$ symmetry). The energy range of these phonon modes (maximum $\sim 400 \mathrm{~cm}^{-1}$ ) is similar to those of CFE (maximum $\sim 450 \mathrm{~cm}^{-1}$ ) and both these excitations are of similar symmetry. Therefore, our observation of renormalization of the phonon modes at low temperature is attributed to the strong coupling with these crystal-field excitations.

Another interesting observation is that the number of possible transitions within the ground state multiplets at low temperature is expected to be only four as shown in the inset of Fig. 5(c); however, seven modes are observed (R1-R7), i.e., three doublets (R1-R2, R3-R4, and R5-R6) and mode R7. At low temperature we observed four strong modes, i.e., $\mathrm{R} 2, \mathrm{R} 4, \mathrm{R} 6$, and R7 and with further decrease in temperature three weak shoulder modes, namely, R1, R3, and R5 also started gaining spectral weight. The frequency difference between these doublets is $\sim 2-3 \mathrm{meV}\left(\sim 20-25 \mathrm{~cm}^{-1}\right)$ and it increases with decrease in temperature [see inset in top panel of Fig. 5(a)]. The observed weak modes, i.e., R1, R3, and R5, may arise from the splitting of the ground state Kramers doublet by the Nd-Nd and Nd-Ir exchange interactions. Interestingly, with increasing temperature the overall intensity of the higher-frequency doublet components (i.e., $\mathrm{R} 2$, R4, and R6) decreases relative to the lower-frequency ones (i.e., R1, R3, and R5) [see Fig. 5(c)]. Also, discontinuities in the intensity follow the long-range magnetic ordering [see inset in top and middle panel in Fig. 5(b)] associated with the two interpenetrating AFM sublattices associated with the $\mathrm{Nd}$ and Ir moments ordering. This discontinuity is also visible in the doublet splitting [see inset in the top panel of Fig. 5(a)]. Quantitatively, the origin of these discontinuities may be understood using the fact that splitting of the Kramers doublets is due to Nd-Nd and Nd-Ir exchange interactions, as these exchange interactions are also responsible for the magnetic ordering, along with a contribution from the dipolar magnetic field due to the Ir ion on the $\mathrm{Nd}$ atom. The observed magnetic transition at $\sim 17 \mathrm{~K}$ roughly corresponds to the exchange interaction strength of $\sim 2 \mathrm{meV}$; therefore the splitting between the ground state Kramers doublet owing to the exchange interactions is expected to be of this order. Our observed splitting of $\sim 25 \mathrm{~cm}^{-1}$ is also close to the value as suggested by the exchange interaction strength. Further, the splitting of the ground state Kramers doublet may also be estimated using the intensity ratio of the doublets and fitting the ratio [see inset in bottom panel of Fig. 5(b)] using the functional form as [41] $I=I_{0} e^{\Delta / k_{b} T}$, where $I_{0}$ is the temperature-independent intensity ratio and $\Delta$ is the splitting of the ground state Kramers doublet. Solid lines in the inset of the bottom panel in Fig. 5(b) shows good agreement between data and fit. The extracted value of the splitting of the ground state Kramers doublet $(\Delta)$ is $\sim 30 \mathrm{~cm}^{-1}$ and is consistent with our observation.

\section{Intraconfigurational transitions and interaction with phonons}

We observed a large number of sharp and strong modes, labeled as Q1-Q46, in the high-energy range spanning from 1700 to $5500 \mathrm{~cm}^{-1}$, shown in Figs. 6(a) and 6(b). The insets show spectra in the absolute energy scale from 17100 to $13300 \mathrm{~cm}^{-1}$. The origin of these sharp modes may be understood as follows. When $\mathrm{Nd}^{3+}$ is placed in $C_{1}$ local site symmetry as in the present case, the ground $\left({ }^{4} I_{9 / 2}\right)$, first excited $\left({ }^{4} I_{11 / 2}\right)$, and second excited $\left({ }^{4} I_{13 / 2}\right)$ state multiplets split into five, six, and seven Kramers doublets, respectively, and so on. In fact, because of the lowest local symmetry at the $\mathrm{Nd}^{3+}$ ions, i.e., $C_{1}$, the degeneracy of the free-ion levels must be completely lifted so that $(2 J+1)$ levels are expected for each $J$ term, which further reduces to $(2 J$ $+1) / 2$ levels as constrained by the Kramers theorem. The doublets of ${ }^{4} G_{5 / 2}+{ }^{2} G_{7 / 2},{ }^{4} F_{9 / 2}$, and ${ }^{4} F_{7 / 2}+{ }^{4} S_{3 / 2}$ are at $\sim 17000 \mathrm{~cm}^{-1}, 14700 \mathrm{~cm}^{-1}$, and $13800 \mathrm{~cm}^{-1}$, respectively $[39,40]$ [see Fig. 6(c) for a schematic of crystal-field splitting of these levels]. Based on the comparative energy with different CF levels, modes from Q1 to Q20 may be assigned to the transitions from higher-lying doublets of $\left({ }^{4} G_{5 / 2}+{ }^{2} G_{7 / 2}\right)$ levels to the different doublets of the ground state $\left({ }^{4} I_{9 / 2}\right)$. Similarly, modes Q21-Q38, and Q39-Q46 may be assigned to transitions from ${ }^{4} F_{9 / 2}$ and ${ }^{4} F_{7 / 2}+{ }^{4} S_{3 / 2}$ to the doublets of the ground state $\left({ }^{4} I_{9 / 2}\right)$, respectively. Figures 6(a) and 6(b) show the modes attributed to the transitions from higher levels to the ground state multiplets, say, from ${ }^{4} G_{5 / 2} /{ }^{2} G_{7 / 2}$ to ${ }^{4} I_{9 / 2}$ in the vicinity of $\sim 2000 \mathrm{~cm}^{-1}\left(\sim 16800 \mathrm{~cm}^{-1}\right.$ in absolute scale). From this figure, we observed a continuous increase in the linewidths, say, as one goes from mode Q6 to Q20; and from Q22 to Q38 (see Table III for the list of prominent mode linewidths with this increasing trend), attributed to the strong electron-phonon interaction. We know that a narrow linewidth is associated with the electronic transitions from the lowest multiplet of any crystal-field level to the lowest level of other multiplets of CF level, and it increases for transitions to the higher multiplets of the same CF level [42]. The underlying mechanism for such a broadening of the linewidth is due to the possible relaxation to the available lower-energy levels with the spontaneous emission of phonons. 



FIG. 6. (a), (b) Raman spectra of $\mathrm{Nd}_{2} \mathrm{ZnIrO}_{6}$ in the frequency range of $1700-4300 \mathrm{~cm}^{-1}$ and $4300-5500 \mathrm{~cm}^{-1}$, respectively, recorded at $75 \mathrm{~K}$ with 532-nm laser excitation; labels Q1-Q46 correspond to intraconfigurational modes (i.e., electronic transition modes of $4 f$ levels of $\mathrm{Nd}^{3+}$ ). The spectra are fitted with a sum of Lorentzian functions, where the solid thick red line indicates a total fit to the experimental data and the solid thin blue line corresponds to individual peak fit. The shaded region shows the same spectra in absolute energy scale. (c) Schematic of the CF levels of $\mathrm{Nd}^{3+}$ ion; vertical blue lines depict the observed transition.

Figures 7(a)-7(c) show the temperature dependence of the prominent modes in the energy range of $13000-18000 \mathrm{~cm}^{-1}$, i.e., Q2, Q5-Q7, Q10, Q11, Q15, Q16, Q22, Q23, Q28, and Q29. The following observations can be made: (i) The frequency of modes Q2, Q5-Q7, Q10, Q11, Q15, Q16, Q22, and Q23 are observed to exhibit significant blueshift with

TABLE III. List of the frequency and linewidth of the prominent intraconfigurational transition modes at $75 \mathrm{~K}$. Units are in $\mathrm{cm}^{-1}$.

\begin{tabular}{|c|c|c|c|c|c|c|c|c|}
\hline \multicolumn{3}{|c|}{${ }^{4} G_{5 / 2}+{ }^{2} G_{7 / 2}$} & \multicolumn{3}{|c|}{${ }^{4} F_{9 / 2}$} & \multicolumn{3}{|c|}{${ }^{4} F_{7 / 2}+{ }^{4} S_{3 / 2}$} \\
\hline Mode & $\omega_{\text {abs. }}$ & $\Gamma$ & Mode & $\omega_{\text {abs. }}$ & $\Gamma$ & Mode & $\omega_{\text {abs. }}$ & $\Gamma$ \\
\hline Q6 & 16875 & 5.2 & Q22 & 14990 & 9.8 & Q39 & 14460 & 16.3 \\
\hline Q7 & 16848 & 10.6 & Q23 & 14938 & 5.8 & Q40 & 14417 & 21.5 \\
\hline Q8 & 16807 & 9.6 & Q24 & 14910 & 8.0 & Q41 & 14360 & 30.1 \\
\hline Q10 & 16756 & 10.4 & Q25 & 14897 & 7.9 & Q42 & 14221 & 40.5 \\
\hline Q11 & 16730 & 15.7 & Q28 & 14827 & 10.7 & Q43 & 14123 & 48.2 \\
\hline Q12 & 16685 & 14.7 & Q29 & 14811 & 12.0 & Q44 & 13885 & 88.5 \\
\hline Q15 & 16620 & 23.4 & Q30 & 14785 & 8.9 & Q45 & 13775 & 132.8 \\
\hline Q16 & 16576 & 11.5 & Q31 & 14777 & 8.9 & Q46 & 13659 & 125.3 \\
\hline Q18 & 16497 & 33.8 & Q33 & 14707 & 20.0 & & & \\
\hline \multirow[t]{3}{*}{ Q20 } & 16399 & 34.7 & Q35 & 14667 & 32.5 & & & \\
\hline & & & Q36 & 14626 & 12.2 & & & \\
\hline & & & Q38 & 14567 & 20.3 & & & \\
\hline
\end{tabular}





FIG. 7. (a), (b) Temperature dependence of frequency and linewidth of intraconfigurational modes. Solid red lines are fitted curves as described in text and solid black and blue lines are guide to the eye. (c) Normalized intensity of the prominent modes Q2, Q5-Q7, Q10, Q11, Q15, Q16, Q22, Q23, Q28, and Q29.

decrease in temperature up to $\sim 100 \mathrm{~K}$; on further decrease in temperature all these modes are redshifted except mode Q10. (ii) Interestingly, the frequency of Q28 and Q29 decreases with decreasing temperature down to $4 \mathrm{~K}$. (iii) The linewidth of all the modes shows line narrowing with decreasing temperature; the decrease in linewidth is as large as $400 \%-500 \%$; for example, see modes Q6, Q7, Q10, and Q11. (iv) The intensity of all the observed modes shows increase with decrease in temperature up to $\sim 100 \mathrm{~K}$, and on further decrease in temperature it decreases for modes Q2, Q5-Q7, Q10, Q22, Q23, and Q29. The intensity of Q11 and Q15 is almost independent of temperature below $\sim 100 \mathrm{~K}$; however, the intensity of modes Q16 and Q28 keeps increasing with decrease in temperature. Temperature evolution of these electronic transitions may be understood by using the fact that the renormalization of the frequencies and their linewidths is intimately linked with the lattice degrees of freedom. Frequency shift is associated with the change in the phonon energy coupled with the transitions, and the expression for the shift in energy for the electronic transition is given as [42]

$$
E_{i}(T)=E_{0 i}+\alpha_{i}\left(\frac{T}{\theta_{D}}\right)^{4} \int_{0}^{\theta_{D} / T} \frac{X^{3}}{e^{X}-1} d X
$$

where $E_{0 i}$ is the energy at $0 \mathrm{~K}, \alpha_{i}=\frac{3}{2} W_{i} \omega_{D}, W_{i}$ is the ionphonon coupling constant, $\omega_{D}$ is the Debye phonon energy, and $\theta_{D}$ is the Debye temperature taken as $\sim 950 \mathrm{~K}$ from our density of phonon calculations. We have fitted the frequency of the prominent mode using the above expression [see solid lines in Figs. 7(a) and 7(b)] and the fitting parameters are given in Table IV. Fitting is in good agreement in the temperature range of 330- 100 K; below $100 \mathrm{~K}$ it starts diverging from the expected behavior. The majority of these modes show a normal temperature dependence of the frequencies, i.e., increase in energy with decreasing temperature, until $\sim 100 \mathrm{~K}$. The temperature evolution may be gauged from the fact that these electronic transitions are mediated by phonons, and they are expected to show similar temperature dependence as that of phonons. From the fitting, the ion-phonon interaction parameter $\left(W_{i}\right)$ is negative for these modes (see Table IV). On the other hand, the temperature dependence of modes Q28 and Q29 is anomalous, i.e., peak frequency decrease with decreasing temperature, also reflected in the positive value of $W_{i}$ for mode Q29. We note that such anomalous temperature evolution has also been observed for different systems [43-45]. 
TABLE IV. List of the parameters obtained from fitting temperature dependence of the prominent intraconfigurational transition modes of $\mathrm{Nd}^{3+}$ in $\mathrm{Nd}_{2} \mathrm{ZnIrO}_{6}$ by using equations as described in the text. Units are in $\mathrm{cm}^{-1} ; W_{i}$ is a dimensionless parameter.

\begin{tabular}{lcrc}
\hline \hline Mode & $\omega(0)$ & $\alpha_{i}$ & $W_{i}$ \\
\hline Q2 & 17042.4 & -102.1 & -0.10 \\
Q5 & 16926.3 & -90.4 & -0.09 \\
Q6 & 16875.5 & -87.3 & -0.08 \\
Q10 & 16755.9 & -31.5 & -0.03 \\
Q11 & 16730.4 & -51.1 & -0.05 \\
Q15 & 16620.6 & -106.3 & -0.11 \\
Q16 & 16575.8 & -50.6 & -0.05 \\
Q22 & 14989.7 & -72.8 & -0.07 \\
Q23 & 14938.5 & -58.9 & -0.06 \\
Q29 & 14810.1 & +57.0 & +0.06 \\
\hline \hline
\end{tabular}

\section{SUMMARY AND CONCLUSION}

In conclusion, anomalous renormalization of the first-order phonon modes below $\sim 100 \mathrm{~K}$ is attributed to the strong coupling between lattice degrees of freedom and crystal-field excitations. The phonon mode frequencies and their symmetries are estimated using first-principle density functional theory based calculations, which are in very good agreement with our polarization-dependent Raman measurements. We observed splitting of ground state Kramers doublets attributed to the exchange interaction between Nd-Nd and Nd-Ir, and the observed doublet splitting is in very good agreement with the estimated values. Our results shed crucial light on the role of $A$-site rare-earth elements in these iridium-based double-perovskite materials reflected via coupled lattice and electronic degrees of freedom, and believe that our results will prove to be an important step in understanding the exotic ground state of these systems by considering electronic and phononic degrees of freedom on equal footing. We hope that our detailed experimental studies revealing a large number of intraconfigurational transitions between $\mathrm{CF}$ levels of $\mathrm{Nd}^{3+}$ at low site symmetry also provide a good starting point to calculate the energy of all possible CF levels in this important class of double-perovskite systems using theoretical models.

\section{ACKNOWLEDGMENTS}

P.K. thanks the Department of Science and Technology, India, for the grant and IIT Mandi for the experimental facilities. The authors at Dresden thank Deutsche Forschungsgemeinschaft (DFG) for financial support via Grant No. DFG AS 523/4-1 (S.A.) and via project B01 of SFB 1143 (Project ID No. 247310070).
[1] Y. Okamoto, M. Nohara, H. Aruga-Katori, and H. Takagi, SpinLiquid State in the $S=1 / 2$ Hyperkagome Antiferromagnet $\mathrm{Na}_{4} \mathrm{Ir}_{3} \mathrm{O}_{8}$, Phys. Rev. Lett. 99, 137207 (2007).

[2] Y. Machida, S. Nakatsuji, S. Onoda, T. Tayama, and T. Sakakibara, Time-reversal symmetry breaking and spontaneous Hall effect without magnetic dipole order, Nature (London, UK) 463, 210 (2010).

[3] S. Nakatsuji, Y. Machida, Y. Maeno, T. Tayama, T. Sakakibara, J. van Duijn, L. Balicas, J. N. Millican, R. T. Macaluso, and J. Y. Chan, Metallic Spin-Liquid Behavior of the Geometrically Frustrated Kondo Lattice $\operatorname{Pr}_{2} \mathrm{Ir}_{2} \mathrm{O}_{7}$, Phys. Rev. Lett. 96, 087204 (2006).

[4] M. K. Crawford, M. A. Subramanian, R. L. Harlow, J. A. Fernandez-Baca, Z. R. Wang, and D. C. Johnston, Structural and magnetic studies of $\mathrm{Sr}_{2} \mathrm{IrO}_{4}$, Phys. Rev. B 49, 9198 (1994).

[5] B. J. Kim, H. Jin, S. J. Moon, J.-Y. Kim, B.-G. Park, C. S. Leem, J. Yu, T. W. Noh, C. Kim, S.-J. Oh, J.-H. Park, V. Durairaj, G. Cao, and E. Rotenberg, Novel $J_{\text {eff }}=1 / 2$ Mott State Induced by Relativistic Spin-Orbit Coupling in $\mathrm{Sr}_{2} \mathrm{IrO}_{4}$, Phys. Rev. Lett. 101, 076402 (2008)

[6] B. J. Kim, H. Ohsumi, T. Komesu, S. Sakai, T. Morita, H. Takagi, and T. Arima, Phase-sensitive observation of a spinorbital Mott state in $\mathrm{Sr}_{2} \mathrm{IrO}_{4}$, Science 323, 1329 (2009).

[7] F. Wang and T. Senthil, Twisted Hubbard Model for $\mathrm{Sr}_{2} \mathrm{IrO}_{4}$ : Magnetism and Possible High Temperature Superconductivity, Phys. Rev. Lett. 106, 136402 (2011).

[8] K.-Y. Yang, Y.-M. Lu, and Y. Ran, Quantum Hall effects in a Weyl semimetal: Possible application in pyrochlore iridates, Phys. Rev. B 84, 075129 (2011).

[9] X. Wan, A. M. Turner, A. Vishwanath, and S. Y. Savrasov, Topological semimetal and Fermi-arc surface states in the elec- tronic structure of pyrochlore iridates, Phys. Rev. B 83, 205101 (2011).

[10] W. Witczak-Krempa and Y. B. Kim, Topological and magnetic phases of interacting electrons in the pyrochlore iridates, Phys. Rev. B 85, 045124 (2012).

[11] D. Pesin and L. Balents, Mott physics and band topology in materials with strong spin-orbit interaction, Nat. Phys. 6, 376 (2010).

[12] B. Singh, G. A. Cansever, T. Dey, A. Maljuk, S. Wurmehl, B. Büchner, and P. Kumar, Orbiton-phonon coupling in $\operatorname{Ir}^{5+}\left(5 d^{4}\right)$ double perovskite $\mathrm{Ba}_{2} \mathrm{YIrO}_{6}$, J. Phys.: Condens. Matter 31, 065603 (2019).

[13] B. Singh, D. Kumar, K. Manna, A. K. Bera, G. A. Cansever, A. Maljuk, S. Wurmehl, B. Büchner, and P. Kumar, Correlated paramagnetism and interplay of magnetic and phononic degrees of freedom in $3 d-5 d$ coupled $\mathrm{La}_{2} \mathrm{CuIrO}_{6}$, J. Phys.: Condens. Matter 31, 485803 (2019).

[14] M. A. Laguna-Marco, P. Kayser, J. A. Alonso, M. J. MartínezLope, M. van Veenendaal, Y. Choi, and D. Haskel, Electronic structure, local magnetism, and spin-orbit effects of Ir(IV)-, Ir(V)-, and Ir(VI)-based compounds, Phys. Rev. B 91, 214433 (2015).

[15] S. Agrestini, K. Chen, C.-Y. Kuo, L. Zhao, H.-J. Lin, C.-T Chen, A. Rogalev, P. Ohresser, T.-S. Chan, S.-C. Weng, G. Auffermann, A. Völzke, A. C. Komarek, K. Yamaura, M. W. Haverkort, Z. Hu, and L. H. Tjeng, Nature of the magnetism of iridium in the double perovskite $\mathrm{Sr}_{2} \mathrm{CoIrO}_{6}$, Phys. Rev. B 100, 014443 (2019).

[16] M. Iakovleva, S. Fuchs, A. Alfonsov, H.-J. Grafe, M. Vogl, S. Aswartham, S. Wurmehl, T. Dey, B. Büchner, E. Vavilova, and V. Kataev, Static and dynamic magnetism of the Ir-based 
double perovskites $\mathrm{La}_{2} \mathrm{BIrO}_{6}(B=\mathrm{Co}, \mathrm{Zn})$ probed by magnetic resonance spectroscopies, Phys. Rev. B 98, 174401 (2018).

[17] M. Vogl, L. T. Corredor, T. Dey, R. Morrow, F. Scaravaggi, A. U. B. Wolter, S. Aswartham, S. Wurmehl, and B. Büchner, Interplay of $3 d$ - and $5 d$-sublattice magnetism in the double perovskite substitution series $\mathrm{La}_{2} \mathrm{Zn}_{1-x} \mathrm{Co}_{x} \mathrm{IrO}_{6}$, Phys. Rev. B 97, 035155 (2018).

[18] P. Kayser, J. M. Martínez-Lope, A. J. Alonso, M. Retuerto, M. Croft, A. Ignatov, and T. M. Fernández-Díaz, Crystal structure, phase transitions, and magnetic properties of iridium perovskites $\mathrm{Sr}_{2} \mathrm{MIrO}_{6}(\mathrm{M}=\mathrm{Ni}, \mathrm{Zn})$, Inorg. Chem. 52, 11013 (2013).

[19] G. Cao, A. Subedi, S. Calder, J.-Q. Yan, J. Yi, Z. Gai, L. Poudel, D. J. Singh, M. D. Lumsden, A. D. Christianson, B. C. Sales, and D. Mandrus, Magnetism and electronic structure of $\mathrm{La}_{2} \mathrm{ZnIrO}_{6}$ and $\mathrm{La}_{2} \mathrm{MgIrO}_{6}$ : Candidate $J_{\text {eff }}=1 / 2$ Mott insulators, Phys. Rev. B 87, 155136 (2013).

[20] A. A. Aczel, A. M. Cook, T. J. Williams, S. Calder, A. D. Christianson, G.-X. Cao, D. Mandrus, Y.-B. Kim, and A. Paramekanti, Highly anisotropic exchange interactions of $j_{\text {eff }}=$ $1 / 2$ iridium moments on the fcc lattice in $\mathrm{La}_{2} B \operatorname{IrO}_{6}(B=\mathrm{Mg}$, Zn), Phys. Rev. B 93, 214426 (2016).

[21] X. Ding, B. Gao, E. Krenkel, C. Dawson, J. C. Eckert, S.-W. Cheong, and V. Zapf, Magnetic properties of double perovskite $L n_{2} \mathrm{CoIrO}_{6}(\mathrm{Ln}=\mathrm{Eu}, \mathrm{Tb}, \mathrm{Ho})$ : Hetero-tri-spin $3 d-5 d-4 f$ systems, Phys. Rev. B 99, 014438 (2019).

[22] M. Vogl, R. Morrow, A. A. Aczel, R. B. Rodriguez, A. U. B. Wolter, S. Wurmehl, S. Aswartham, and B. Büchner, Complex magnetic properties in the mixed $4 f-5 d$ double perovskite iridates $L n_{2} \mathrm{ZnIrO}_{6}(L n=\mathrm{Nd}, \mathrm{Sm}, \mathrm{Eu} \& \mathrm{Gd})$, arXiv:1910.13552.

[23] A. A. Aczel, J. P. Clancy, Q. Chen, H. D. Zhou, D. Reig-iPlessis, G. J. MacDougall, J. P. C. Ruff, M. H. Upton, Z. Islam, T. J. Williams, S. Calder, and J.-Q. Yan, Revisiting the Kitaev material candidacy of $\mathrm{Ir}^{4+}$ double perovskite iridates, Phys. Rev. B 99, 134417 (2019).

[24] G. Jackeli and G. Khaliullin, Mott Insulators in the Strong SpinOrbit Coupling Limit: From Heisenberg to a Quantum Compass and Kitaev Models, Phys. Rev. Lett. 102, 017205 (2009).

[25] B. Singh, M. Vogl, S. Wurmehl, S. Aswartham, B. Büchner, and P. Kumar, Kitaev magnetism and fractionalized excitations in double perovskite $\mathrm{Sm}_{2} \mathrm{ZnIrO}_{6}$, Phys. Rev. Res. 2, 013040 (2020).

[26] S. Jandl, P. Dufour, T. Strach, T. Ruf, M. Cardona, V. Nekvasil, C. Chen, and B. M. Wanklyn, Raman study of intermultiplet crystal-field excitations in $\mathrm{Nd}_{2} \mathrm{CuO}_{4}$, Phys. Rev. B 52, 15558 (1995).

[27] S. Taboada, A. de Andrés, J. E. Muñoz Santiuste, C. Prieto, J. L. Martínez, and A. Criado, Optical phonons, crystal-field transitions, and europium luminescence-excitation processes in $\mathrm{Eu}_{2} \mathrm{BaCoO}_{5}$ : Experiment and theory, Phys. Rev. B 50, 9157 (1994).

[28] P. Kumar, A. Kumar, S. Saha, D. V. S. Muthu, J. Prakash, U. V. Waghmare, A. K. Ganguli, and A. K. Sood, Temperaturedependent Raman study of a $\mathrm{CeFeAsO} \mathrm{O}_{0.9} \mathrm{~F}_{0.1}$ superconductor: crystal field excitations, phonons and their coupling, J. Phys.: Condens. Matter 22, 255402 (2010).

[29] P. Kumar, D. V. S. Muthu, L. Harnagea, S. Wurmehl, B. Büchner, and A. K. Sood, Phonon anomalies, orbital- ordering and electronic Raman scattering in iron-pnictide $\mathrm{Ca}\left(\mathrm{Fe}_{0.97} \mathrm{Co}_{0.03}\right)_{2} \mathrm{As}_{2}$ : temperature-dependent Raman study, J. Phys.: Condens. Matter 26, 305403 (2014).

[30] G. Schaack, Raman scattering by crystal-field excitations, in Light Scattering in Solids VII: Crystal-Field and Magnetic Excitation, Topics in Applied Physics Vol. 75, edited by M. Cardona and G. Güntherodt (Springer, Berlin, 2000).

[31] P. Giannozzi, S. Baroni, N. Bonini, M. Calandra, R. Car, C. Cavazzoni, D. Ceresoli, G. L. Chiarotti, M. Cococcioni, and I. Dabo et al., QUANTUM ESPRESSO: a modular and open-source software project for quantum simulations of materials, J. Phys.: Condens. Matter 21, 395502 (2009).

[32] P. Giannozzi, S. de Gironcoli, P. Pavone, and S. Baroni, Ab initio calculation of phonon dispersions in semiconductors, Phys. Rev. B 43, 7231 (1991).

[33] J. P. Perdew, A. Ruzsinszky, G. I. Csonka, O. A. Vydrov, G. E. Scuseria, L. A. Constantin, X. Zhou, and K. Burke, Restoring the Density-Gradient Expansion for Exchange in Solids and Surfaces, Phys. Rev. Lett. 100, 136406 (2008).

[34] M. Balkanski, R. F. Wallis, and E. Haro, Anharmonic effects in light scattering due to optical phonons in silicon, Phys. Rev. B 28, 1928 (1983).

[35] E. Granado, A. García, J. A. Sanjurjo, C. Rettori, I. Torriani, F. Prado, R. D. Sánchez, A. Caneiro, and S. B. Oseroff, Magnetic ordering effects in the Raman spectra of $\mathrm{La}_{1-x} \mathrm{Mn}_{1-x} \mathrm{O}_{3}$, Phys. Rev. B 60, 11879 (1999).

[36] L. H. Hall, Group Theory and Symmetry in Chemistry (McGrawHill Inc., New York, 1969).

[37] R. Loudon, The Raman effect in crystals, Adv. Phys. 13, 423 (1964).

[38] D. Kumar, B. Singh, R. Kumar, M. Kumar, and P. Kumar, Anisotropic electron-photon-phonon coupling in layered $\mathrm{MoS}_{2}$, arXiv:2001.04202.

[39] M. N. Popova, E. P. Chukalina, T. N. Stanislavchuk, B. Z. Malkin, A. R. Zakirov, E. Antic-Fidancev, E. A. Popova, L. N. Bezmaternykh, and V. L. Temerov, Optical spectra, crystalfield parameters, and magnetic susceptibility of multiferroic $\mathrm{NdFe}_{3}\left(\mathrm{BO}_{3}\right)_{4}$, Phys. Rev. B 75, 224435 (2007).

[40] A. Mech, Z. Gajek, M. Karbowiak, and C. Rudowicz, Crystalfield energy level analysis for $\mathrm{Nd}^{3+}$ ions at the low symmetry $C_{1}$ site in $\left[\mathrm{Nd}(\mathrm{hfa})_{4}\left(\mathrm{H}_{2} \mathrm{O}\right)\right]\left(\mathrm{N}\left(\mathrm{C}_{2} \mathrm{H}_{5}\right)_{4}\right)$ single crystals, J. Phys.: Condens. Matter 20, 385205 (2008).

[41] P. Dufour, S. Jandl, C. Thomsen, M. Cardona, B. M. Wanklyn, and C. Changkang, Raman study of Kramers doublets in $\mathrm{Nd}_{2} \mathrm{CuO}_{4}$, Phys. Rev. B 51, 1053 (1995).

[42] S. Hiifner, Optical Spectra of Transparent Rare Earth Compounds (Academic Press, New York, 1978).

[43] T. Kushida, Linewidths and thermal shifts of spectral lines in neodymium-doped yttrium aluminum garnet and calcium fluorophosphate, Phys. Rev. 185, 500 (1969).

[44] S. Saha, S. Prusty, S. Singh, R. Suryanarayanan, A. Revcolevschi, and A. K. Sood, Anomalous temperature dependence of phonons and photoluminescence bands in pyrochlore $\mathrm{Er}_{2} \mathrm{Ti}_{2} \mathrm{O}_{7}$ : signatures of structural deformation at $130 \mathrm{~K}, \mathrm{~J}$. Phys.: Condens. Matter 23, 445402 (2011).

[45] M. G. Beghi, C. E. Bottani, and V. Russo, Debye temperature of erbium-doped yttrium aluminum garnet from luminescence and Brillouin scattering data, J. Appl. Phys. 87, 1769 (2000). 